0417 PRELIMINARY STUDY OF DISTRESS AMONG MEDICAL AND PARAMEDICAL STAFF OF MOROCCAN HOSPITAL

'Fatima-Zahra Azzaoui, ${ }^{1}$ Hassan Chtibi, ${ }^{1}$ Ahmed Ahami, ${ }^{2}$ Hinde Hami. ${ }^{1}$ Equip of Clinical and Cognitive Neurosciences and Health, Faculty of Science, Kenitra, Morocco; ${ }^{2}$ Laboratory of Genetics and Biometry, Faculty of Sciences, Kenitra, Morocco

\subsection{6/oemed-2014-102362.367}

Objectives Mental health status of medical and paramedical staff has an impact on the quality and quantity of their productivity and on their relationship with the patients.

The objective of this preliminary study is to measure mental health status in hospital staff, especially, emotional disorder "distress".

Method The study is realised in hospital in Rabat, Morocco, among 100 medical and paramedical staff. The 12-items General Health Questionnaire GHQ) and questionnaire about their health status are used.

Results The results show that, according to gender; among 50 men, 50\% suffer from distress and among 50 women, $58 \%$ are distressed.

Also, according to specialty; $80 \%$ of doctors and $47.5 \%$ of nurses in different categories $55 \%$ of nurses in anaesthesia and resuscitation, $45 \%$ of nurses of laboratory, polyvalent nurses and radiology nurses) suffer from this emotional disorder.

The findings demonstrate also, that $23 \%$ and $36 \%$ of the staff sample's suffer from different health problems (gastritis, allergies, asthma, colopathy, hypertension...) and from sleep disorders respectively.

Conclusions These preliminary results have highlighted the danger that threatens the health status of medical and paramedical staff of this hospital. Deeper investigations are needed to determine all the possible factors that could be influencing this status and studying the possible relationship between the founded pathologies and distress.

\section{SEVERE OCCUPATIONAL INJURIES IN MOROCCO}

${ }^{1}$ Hinde Hami, ${ }^{2}$ Fatima-Zahra Azzaoui, ${ }^{2}$ Driss Raougui, ${ }^{2}$ Ahmed Omar Touhami Ahami. 'Laboratory of Genetics and Biometry, Faculty of Science, Ibn Tofail University, Kenitra, Morocco; ${ }^{2}$ Equip of Clinic and Cognitive Neurosciences and Health, Laboratory of Biology and Health, Faculty of Science, Ibn Tofail University, Kenitra, Morocco

\subsection{6/oemed-2014-102362.368}

Objectives Occupational injuries represent a considerable part of the injury burden to society, affecting people in the most productive years of their lives. The aim of this study is to describe the profile of severe occupational injuries in Kenitra city, economic capital of the Gharb region (NW Morocco).

Method This is a descriptive retrospective analysis of severe occupational injuries (fatal injuries or resulting in permanent disability) notified in the delegation of employment of Kenitra in 2008-2009. The results do not include occupational diseases or journey accidents.

Results There were 210 severe workplace injuries reported; 176 resulted in temporary disability and 34 were fatal. According to data recorded, $91 \%$ of the victims were men with a male-female ratio of 10.05 . The most exposed sectors were building and public works sector (38\%), wood, furniture, paper, cardboard, textile and clothing industries (29.5\%) and metallurgical industries (10\%). Accidents were caused by machinery and falling materials, followed by falls from height and electricity.
Conclusions The assessment and prevention of occupational risks are a major asset to improve the quality of work and retain employees through a better quality of work life.

\section{DEVELOPMENT OF A DISEASE SURVEILLANCE SYSTEM FOR SILICOSIS AND RESPIRATORY DISORDERS IN STONE CARVING WORKERS IN THAILAND}

Krittin Silanun. Faculty of Medicine, Khon Kaen University, Thailand

\subsection{6/oemed-2014-102362.369}

Objectives To (a) develop a surveillance system for silicosis (b) estimate prevalence of silicosis and respiratory disorders among stone carversand (c) develop a guideline for screening for silicosis.

Method This was a cross-sectional, descriptive study of 1257 stone carvers. Data were collected between July and October, 2012. The 767 participants in the study were classified by job categories. Exposure indices were constructed. Health outcomes (including job description, respiratory symptoms and chest radiographs) were assessed and confirmed bydiagnosis by a B reader. Results Of the total population, 767 underwent chest radiographs (age24 to 75 years; 97.1\% male). The duration of exposure was between 2 and 30 years. The prevalence of radiographic change was $8.9 \%$ (68 subjects). There were 66 subjects with parenchymal lesions and profusion $(>$ grade $1 / 0$ as per ILO classification). Two subjects have pleural abnormalities. Importantly, 55 cases among 68 with radiographic abnormalitieswere compatible with tuberculosis; 32 of whom showed no clinical evidence of tuberculosis.

Conclusions The diagnostic differentiation between silicosis and tuberculosis ischallenging; consequently, discrepancies can arise when reporting the prevalence of the two diseases. Our research group isdeveloping CPG for screening silicosis forreferral to a clinician in chest medicine. The remaining at-risk population will be examined by chest X-ray in July 2013 and the hazard surveillance and exposure to silica performed next.

\section{OCCUPATION AND LEUKAEMIA IN SPAIN 2007-2012}

${ }^{1}$ Marta M Rodríguez, ${ }^{2}$ Ana Fernández-Somoano, ${ }^{3} J u a n$ Alguacil, ${ }^{4}$ Miguel Santibañez, ${ }^{5}$ Gemma Castano, ${ }^{6}$ Javier Llorca, ${ }^{7}$ Rafael Marcos, ${ }^{5}$ Manolis Kogevinas, ${ }^{7}$ Silvia Sanjose, ${ }^{2}$ Adonina Tardon. ${ }^{1}$ Health Service SESPA, Oviedo, Asturias, Spain; ${ }^{2}$ Molecular Epidemiology of Cancer Unit, University Institute of Oncology, University of Oviedo, Asturias, Spain; ${ }^{3}$ Universidad de Huelva, Huelva, Spain; ${ }^{4}$ Universidad de Cantabria, Cantabria, Spain; ${ }^{5}$ Centre for Research in Environmental Epidemiology, Barcelona, Cataluña, Spain; ${ }^{6}$ Division of Preventive Medicine and Public Health, University of Cantabria, Cantabria, Spain; ${ }^{7}$ Institut Català d'Oncologia, Girona, Spain

\subsection{6/oemed-2014-102362.370}

Objectives Established risk factors for leukaemia do not explain the majority of leukaemia. Previous studies have suggested the importance of occupation in leukaemogenesis.

To evaluate associations between job title and leukaemia in the population the MCC-Spain

We studied occupational variation of the risk of chronic lymphocytic leukaemia

Method We have 30744 occupational interviews recruited during 2007 to 2012 all was codified on 67 group homogeneous units, according to a defined criteria, in the same category defined by a set of tasks of the same characteristics. We analysed 196 cases of leukaemia (aged 20-75 years) and yours controls 